\title{
Evidence for Nonlinear Asymmetric Causality in US Inflation, Metal, and Stock Returns
}

\author{
D. Hristu-Varsakelis ${ }^{1}$ and C. Kyrtsou ${ }^{2}$ \\ ${ }^{1}$ Department of Applied Informatics, University of Macedonia, 156 Egnatia Street, \\ Thessaloniki 54006, Greece \\ ${ }^{2}$ Department of Economics, University of Macedonia, 156 Egnatia Street, Thessaloniki 54006, Greece
}

Correspondence should be addressed to C. Kyrtsou, ckyrtsou@uom.gr

Received 2 October 2007; Accepted 9 May 2008

Recommended by Masahiro Yabuta

The purpose of this paper is to propose a version of causality testing that focuses on how the sign of the returns affects the causality results. We replace the traditional VAR specification used in the Granger causality test by a discrete-time bivariate noisy Mackey glass model. Our test reveals interesting and previously unexplored relationships in US economic series, including inflation, metal, and stock returns.

Copyright (C) 2008 D. Hristu-Varsakelis and C. Kyrtsou. This is an open access article distributed under the Creative Commons Attribution License, which permits unrestricted use, distribution, and reproduction in any medium, provided the original work is properly cited.

\section{Introduction}

Since the concept of Granger causality was first proposed [1], there has been a growing body of research devoted on the subject of causal relationships between economic variables. Among the central goals of that research are (i) the elucidation of the connections that exist between different components of an economic system, with all its complexity and (ii) the use of causality links (where detected) in designing efficient economic policies. Granger's pioneering work on linear causality has been extended and adapted to take into account nonlinear dependencies in the quantities studied, as in $[2,3]$ and more recently [4, 5], among others.

The contribution of this paper is to propose a nonlinear version of Granger causality test, which conditions on samples of the causing series being positive or negative. This "asymmetric" causality test, which builds on [6], is a way to "sharpen" the usual version of causality testing in which the series being tested are considered in their entirety, all at once. Asymmetric causality testing can reveal interesting information about the inherent dynamics of the processes studied. For example, detection of a causality relationship does not give information on whether a positive or negative shock has significant predictive value. 
Conversely, lack of an apparent causality relationship does not preclude the existence of causality when we condition for certain features, such as a positive or negative sign.

The remainder of this paper is organized as follows. In Section 2, we describe a model to be used for studying causality relationships, together with a Granger-type asymmetric causality test. We replace the linear VAR specification used by Granger by a discrete-time, noisy Mackey-Glass model, in order to detect nonlinear "links" between the variables studied. The choice of model is not unique and was guided by the recently investigated ability of the model to reproduce properties of real economic data [7-9]. The deterministic Mackey-Glass equation has also been used in the neural network literature [10,11]. Section 3 discusses the application of our test in real economic series which include data on US inflation, as well as metal and stock returns.

\section{A test for nonlinear asymmetric causality}

The causality test proposed in this paper extends the test for nonlinear Granger causality introduced in [6]. In its symmetric version, it assumes an underlying process with a special type of nonlinear structure, known as the bivariate noisy Mackey-Glass (hereafter "M-G") model [7]. The model is as follows:

$$
\begin{aligned}
& X_{t}=\alpha_{11} \frac{X_{t-\tau_{1}}}{1+X_{t-\tau_{1}}^{c_{1}}}-\delta_{11} X_{t-1}+\alpha_{12} \frac{Y_{t-\tau_{2}}}{1+Y_{t-\tau_{2}}^{c_{2}}}-\delta_{12} Y_{t-1}+\epsilon_{t} \quad \epsilon_{t} \sim N(0,1), \\
& Y_{t}=\alpha_{21} \frac{X_{t-\tau_{1}}}{1+X_{t-\tau_{1}}^{c_{1}}}-\delta_{21} X_{t-1}-\alpha_{22} \frac{Y_{t-\tau_{2}}}{1+Y_{t-\tau_{2}}^{c_{2}}}-\delta_{22} Y_{t-1}+u_{t} \quad u_{t} \sim N(0,1) \text {, }
\end{aligned}
$$

where $t=\tau, \ldots, N, \tau=\max \left(\tau_{1}, \tau_{2}\right)$ and $X_{0}, \ldots, X_{\tau-1}, Y_{0}, \ldots, Y_{\tau-1}$ are given. The $\alpha_{i j}$, and $\delta_{i j}$ are parameters to be estimated, $\tau_{i}$ are integer delays, and $c_{i}$ are constants. The model (2.1) can produce various types of dependencies by adjusting the parameters $c_{i}$ and $\tau_{i}$. The principal advantage of (2.1) over simple VAR alternatives is that the M-G terms are able to capture more complex dependent dynamics in a time series. As discussed in $[7,12]$, the presence of statistically significant $M-G$ terms in a pair of series suggests nonlinear feedback as the generating mechanism of the interdependences between $X$ and $Y$.

The M-G-based causality test attempts to detect whether past samples of a variable $Y$ have a significant nonlinear effect (of the type $Y_{t-\tau_{2}} /\left(1+Y_{t-\tau_{2}}^{c_{2}}\right)$ ) on the current value of another variable $X$. Operationally, the test is similar to the linear Granger causality test, except that the models fitted to the series are M-G processes. One begins by estimating the parameters of an M-G model that best fits the given series, using ordinary least squares. To test for causality from $Y$ to $X$, a second M-G model is estimated, under the constraint $\alpha_{12}=0$. The latter equation is our null hypothesis. Let $\widehat{\epsilon}_{t}, \widehat{\theta}_{t}$ be the residuals produced by the unconstrained and constrained best-fit M-G models, respectively. We compute and compare the sums of squared residuals

$S_{c}=\sum_{t=1}^{N} \widehat{\theta}_{t}$ and $S_{u}=\sum_{t=1}^{N} \widehat{\epsilon}_{t}$. Let $n_{\text {free }}=4$ be the number of free parameters in our M-G model and $n_{\text {rest } r}=1$ be the number of parameters set to zero when estimating the constrained model. If the test statistic

$$
S_{F}=\frac{\left(S_{c}-S_{u}\right) / n_{\text {rest } r}}{S_{u} /\left(N-n_{\text {free }}-1\right)} \sim F_{n_{\text {rest } r}, N-n_{\text {free }}-1}
$$


is greater than a specified value, then we reject the null hypothesis that $Y$ causes $X$ (recall that $S_{F}$ follows an $F$ distribution under the null hypothesis.) The $p$-value for the test is computed from

$$
p=1-F_{n_{\text {rest } r}, N-n_{\text {free }-1}}^{\mathrm{cdf}}\left(S_{F}, n_{\text {rest } r}, N-n_{\text {free }}-1\right),
$$

where $F_{a, b}^{c d f}$ is the cumulative distribution function for the $F_{a, b}$ distribution. The test requires prior selection of the parameters of the M-G process, namely, $\tau_{1}, \tau_{2}, c_{1}, c_{2}$. The best delays $\tau_{1}, \tau_{2}$, are to be chosen on the basis of likelihood ratio tests and the Schwarz criterion. We will refer to the above test as "symmetric" to distinguish it from its specialized counterpart described next.

Regardless of the causality relationships identified in the pair $X$ and $Y$, we would like to know whether those relationships hold when conditioning for positive or negative returns. For example, one may want to know whether positive returns observed in one series give predictive information that is more significant than that given by negative returns; alternatively, there may be settings in which causality exists only in a subset of the observations, (e.g., for positive returns). To investigate such relationships, we consider an "asymmetric" causality test that conditions on the causing series being nonnegative or nonpositive. Briefly, the asymmetric test is based on the M-G test described above, where the best M-G model is calculated using only those observations that satisfy the appropriate condition on the returns. To make matters concrete, suppose that in model (2.1) we would like to test whether nonnegative returns in the series $Y$ cause the series $X$. An observation $\left(X_{i}, Y_{i}\right)$ is included for regression only if $Y\left(t-\tau_{2}\right) \geq 0$. The same restricted set of observations is used to compute the model corresponding to the null hypothesis (in this case $\alpha_{12}=0$ ). The procedure is then repeated, with the order of the series reversed (to investigate whether positive returns in $X$ cause $Y$ ) and again with the subset of observations that correspond to nonpositive returns.

In this work, we have chosen to condition on the sign of the causing series because of its practical relevance. Of course, nonpositivity (resp., nonnegativity) is by no means the only type of conditioning possible, so that in addition to varying the choice of dynamics fitted to the series, one can test for causality given various other events (e.g., start/end of the week, price movement thresholds, etc.).

\section{Results and discussion}

We applied our test to two pairs of series. The first pair included US consumer price (CPI) and metal price (MET) indices. The consumer price index data are from the BLS CPI-all urban consumers CUUROOOOAA0 index, reported in Free Lunch. BLS producer price index WPU10 for metals and metal products from the BLS website. In the second pair, the metal price index was replaced by the Dow-Jones stock price index (PSDJ). The Dow Jones Stock Prices Index includes the NBER series \#11009 representing 20 stocks until 1928, and 30 stocks thereafter, ending on 12/1968. Beginning in 01/1969, it is updated from the Dow Jones Index for Industrials: 30 Industries (index $1920=100$, monthly end) -reported in Free Lunch. The series contained monthly observations from 01/1960 to 07/2002 (511 obs.) and were not seasonally adjusted. That metal price index was selected because it is one of the commodity price series having a significant impact on manufacturing activities, and hence inflation. Prior to causality testing, we performed the Dickey-Fuller test $[13,14]$ to identify unit roots in all 
Table 1: Test for nonlinear causality (symmetric case). If $P>.05$, then at $5 \%$ we accept the null hypothesis that $A$ does not cause $B$. The parameters for the M-G model were $\tau_{1}=3, \tau_{2}=1$, and $c_{1}=c_{2}=2$.

\begin{tabular}{lcc}
\hline Relation $(A \rightarrow B)$ & F-statistic & Probability \\
\hline DLCPI $\rightarrow$ DLMET & 4.5745 & 0.0329 \\
DLMET $\rightarrow$ DLCPI & 3.9202 & 0.0483 \\
\hline
\end{tabular}

Table 2: Test for nonlinear causality (asymmetric case for positive returns). If $P>.05$, then at $5 \%$ we accept the null hypothesis that $A$ does not cause $B$. The parameters for the M-G model were $\tau_{1}=3, \tau_{2}=1$, and $c_{1}=c_{2}=2$.

\begin{tabular}{lcc}
\hline Relation $(A \rightarrow B)$ & $F$-statistic & Probability \\
\hline $\mathrm{DLCPI}^{p} \rightarrow$ DLMET & 2.6389 & 0.1049 \\
DLMET $^{p} \rightarrow$ DLCPI & 18.9806 & $1.6 \mathrm{E}-5$ \\
\hline
\end{tabular}

Table 3: Test for nonlinear causality (asymmetric case for negative returns). If $P>.05$, then at $5 \%$ we accept the null hypothesis that $A$ does not cause $B$. The parameters for the M-G model were $\tau_{1}=3, \tau_{2}=1$, and $c_{1}=c_{2}=2$.

\begin{tabular}{lcc}
\hline Relation $(A \rightarrow B)$ & $F$-statistic & Probability \\
\hline $\mathrm{DLCPI}^{n} \rightarrow$ DLMET & 5.9587 & 0.0150 \\
$\mathrm{DLMET}^{n} \rightarrow$ DLCPI & 4.1406 & 0.0424 \\
\hline
\end{tabular}

series. For each series CPI, MET, and PSDJ, the test showed that a unit root was present, therefore the log-differenced version of the series was used, giving us DLCPI, DLMET, and DLPSDJ, respectively.

\subsection{Series DLCPI, DLMET}

Table 1 shows the results of our symmetric causality test on the series CPI and MET. The test identified bidirectional causality, confirming the nonlinear feedback mechanism proposed in $[12,15]$. To find out whether specifying the direction of series movement has a significant effect on the causality relationships identified, we performed the asymmetric causality test on the same series. The results are shown in Tables 2 and 3. The exponent " $p$ " (resp., " $n$ ") indicates that only the positive (resp., negative) values of a series were included. The only relationship that differs qualitatively from what is identified in the symmetric test is the lack of M-G feedback between DLCIP ${ }^{p}$ and DLMET. Of course, we cannot exclude other possible forms of nonlinear causality, however, based on the M-G structure adopted here, the causality from DLCPI to DLMET is mainly due to the predictive information contained in the negative DLCPI movements on the DLMET series. We also note that, based on Table 1 only, one could assert that DLCPI causes DLMET. That statement would be in doubt, however, in the event that CPI movement was positive, as Tables 2 and 3 show. The fact that positive MET returns strongly affect inflation suggests that one should consider more seriously the impact of asymmetries in commodity price behavior. As highlighted in [15], the implications of such relationships can be of profound interest if we take into account the fact that traditional US policy relies heavily on monetary policy to control inflation effects. 
Table 4: Test for nonlinear causality (symmetric case). If $P>.05$, then at $5 \%$ we accept the null hypothesis that $A$ does not cause $B$. The M-G model parameters were $\tau_{1}=\tau_{2}=1$, and $c_{1}=c_{2}=2$.

\begin{tabular}{lcc}
\hline Relation $(A \rightarrow B)$ & $F$-statistic & Probability \\
\hline DLCPI $\rightarrow$ DLPSDJ & 0.2014 & 0.6538 \\
DLPSDJ $\rightarrow$ DLCPI & 0.3002 & 0.5840 \\
\hline
\end{tabular}

Table 5: Test for nonlinear causality (asymmetric case for positive returns). If $P>.05$, then at $5 \%$ we accept the null hypothesis that $A$ does not cause $B$. The M-G model parameters were $\tau_{1}=\tau_{2}=1$, and $c_{1}=c_{2}=2$.

\begin{tabular}{lcc}
\hline Relation $(A \rightarrow B)$ & $F$-statistic & Probability \\
\hline DLCPI $^{p} \rightarrow$ DLPSDJ & 0.4780 & 0.4896 \\
DLPSDJ $^{p} \rightarrow$ DLCPI & 19.7506 & $1.0853 \mathrm{E}-5$ \\
\hline
\end{tabular}

Table 6: Test for nonlinear causality (asymmetric case for negative returns). If $P>.05$, then at $5 \%$ we accept the null hypothesis that $A$ does not cause $B$. The M-G model parameters were $\tau_{1}=\tau_{2}=1$, and $c_{1}=c_{2}=2$.

\begin{tabular}{lcc}
\hline Relation $(A \rightarrow B)$ & $F$-statistic & Probability \\
\hline DLCPI $^{n} \rightarrow$ DLPSDJ & 53.9845 & $8.1768 \mathrm{E}-13$ \\
DLPSDJ $^{n} \rightarrow$ DLCPI & 3.7390 & 0.0537 \\
\hline
\end{tabular}

\subsection{Series DLCPI, DLPSDJ}

Table 4 shows the results of our symmetric causality test on the series CPI and PSDJ. The test did not identify M-G causality. When we conditioned on the causing series being positive or negative, strong unidirectional M-G causality from DLPSDJ ${ }^{p}$ to DLCPI and from DLCPI ${ }^{n}$ to DLPSDJ was revealed (Tables 5 and 6, resp.).

Although the results of our test do not preclude the existence of other models that "explain" the series interdependence, they do confirm that the M-G model has significant predictive power when we take into account positive values of PSDJ or negative values of DLCPI. Furthermore, the coefficients in the best-fit M-G model indicate a positive relation between DLPSDJ ${ }^{p}$ and DLCPI (meaning that the coefficients of both DLPSDJ ${ }^{p}$ terms in the DLCPI equation were positive). This is in line with the empirical findings in [16] which identified a positive relationship between stock returns and inflation, brought about by demand shocks (e.g., money supply fluctuations). The coefficients of the DLCPI ${ }^{n}$ terms in the DLPSDJ equation were both negative, indicating a negative relation between DLCPI ${ }^{n}$ and DLPSDJ. This agrees with the explanation advanced in [17] that inflation may indirectly be responsible for negative fluctuations in stock returns via real output movements, when supply shocks perturb the market.

\section{Conclusions}

This paper proposed an asymmetric version of a nonlinear (Mackey-Glass) causality test introduced in [6]. The test attempts to detect causality relationships in pairs of economic series, conditioning on the sign (negative or positive) of returns of the causing series. We have demonstrated the use of our test in data concerning US inflation, metal, and stock prices. For the relationship between inflation and metal returns series, we have found that 
the bidirectional causality identified is mainly due to positive movements of metal prices and negative movements of inflation. For inflation and stock returns, we found strong evidence of unidirectional causality, from positive stock returns and negative inflation. Our test confirms existing economics-based explanations of the relationships between the series studied. The application of conditioning to reveal "asymmetric" causality is useful for (i) refining the origins of detected causality and (ii) detecting nonlinear feedback structures when no such structure is apparent in the original series. In this work, we used the M-G process to expose an interesting relationship between the three variables studied, however, one could consider other (non-MG) dynamics, as well as asymmetric versions of other causality tests, such as [2, 3, 18]. Ongoing work includes an investigation of our test via Monte Carlo experiments, in order to explore its properties with samples which are significantly smaller than the ones used in this work.

\section{Acknowledgment}

The authors would like to thank the anonymous reviewers for their helpful comments and suggestions.

\section{References}

[1] C. W. J. Granger, "Investigating causal relations by econometric methods and cross-spectral methods," Econometrica, vol. 37, no. 3, pp. 424-438, 1969.

[2] C. Hiemstra and J. Jones, "Testing for linear and nonlinear Granger causality in the stock price-volume relation," The Journal of Finance, vol. 49, no. 5, pp. 1639-1664, 1994.

[3] C. Diks and V. Panchenko, "A note on the Hiemstra-Jones test for Granger non-causality," Studies in Nonlinear Dynamics \& Econometrics, vol. 9, no. 2, article 4, 7 pages, 2005.

[4] C. Gouriéroux and J. Jasiak, "Nonlinear causality with applications to liquidity and stochastic volatility," submitted to Journal of Economic Theory

[5] A. Peguin-Feissolle, B. Strikholm, and T. Teräsvirta, "Testing the Granger noncausality hypothesis in stationary nonlinear models of unknown functional form," Working Paper Series in Economics and Finance 672, Stockholm School of Economics, Stockholm, Sweden, 2007.

[6] D. Hristu-Varsakelis and C. Kyrtsou, "Testing for Granger causality in the presence of chaotic dynamics," to appear in Brussels Economic Review.

[7] C. Kyrtsou and W. C. Labys, "Evidence for chaotic dependence between US inflation and commodity prices," Journal of Macroeconomics, vol. 28, no. 1, pp. 256-266, 2006.

[8] C. Kyrtsou and A. Serletis, "Univariate tests for nonlinear structure," Journal of Macroeconomics, vol. 28, no. 1, pp. 154-168, 2006.

[9] C. Kyrtsou and M. Terraza, "Is it possible to study chaotic and ARCH behaviour jointly? Application of a noisy Mackey-Glass equation with heteroskedastic errors to the Paris Stock exchange returns series," Computational Economics, vol. 21, no. 3, pp. 257-276, 2003.

[10] R. A. Gallant and H. White, "On learning the derivatives of an unknown mapping with multilayer feedforward networks," in Artificial Neural Networks, pp. 206-233, Blackwell, Oxford, UK, 1992.

[11] W. L. Goffe, G. D. Ferrier, and J. Rogers, "Global optimization of statistical functions with simulated annealing," Journal of Econometrics, vol. 60, no. 1-2, pp. 65-99, 1994.

[12] C. Kyrtsou and W. C. Labys, "Detecting positive feedback in multivariate time series: the case of metal prices and US inflation," Physica A, vol. 377, no. 1, pp. 227-229, 2007.

[13] D. A. Dickey and W. A. Fuller, "Distribution of the estimators for autoregressive time series with a unit root," Journal of the American Statistical Association, vol. 74, no. 366, part 1, pp. 427-431, 1979.

[14] D. A. Dickey and W. A. Fuller, "Likelihood ratio statistics for autoregressive time series with a unit root," Econometrica, vol. 49, no. 4, pp. 1057-1072, 1981.

[15] C. Kyrtsou, "Nonlinear features of comovements between commodity prices and inflation," in Proceedings of the Festschrift Symposium for Walter C. Labys, Agricaltural and Resource Economics, West Virginia University, Morgantown, WVa, USA, May 2007. 
[16] P. J. Hess and B.-S. Lee, "Stock returns and inflation with supply and demand disturbances," The Review of Financial Studies, vol. 12, no. 5, pp. 1203-1218, 1999.

[17] S. Karagianni and C. Kyrtsou, "Analysing and dynamics between US inflation and dow jones index using non-linear methods," Studies in Nonlinear Dynamics \& Econometrics, under review.

[18] W.-X. Zhou and D. Sornette, "Non-parametric determination of real-time lag structure between two time series: the "optimal thermal causal path" method with applications to economic data," Journal of Macroeconomics, vol. 28, no. 1, pp. 195-224, 2006. 


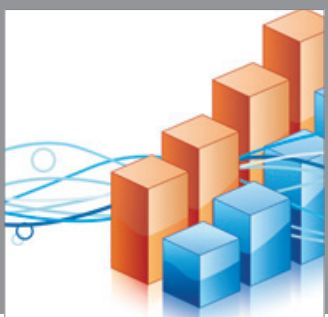

Advances in

Operations Research

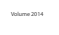

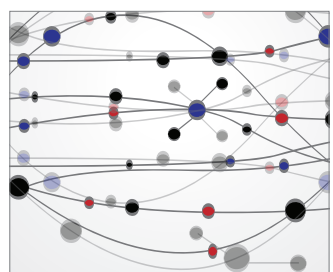

\section{The Scientific} World Journal
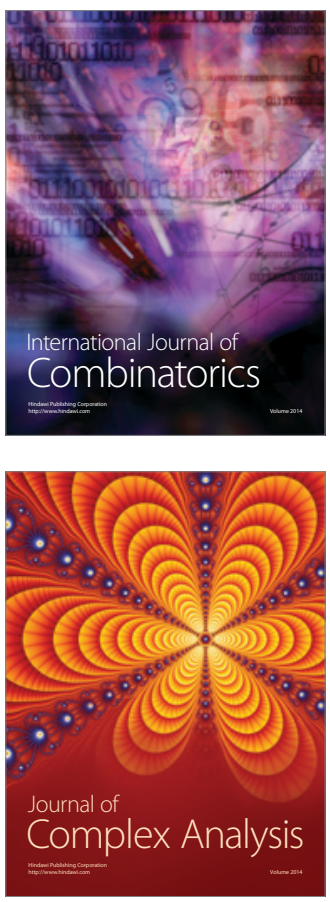

International Journal of

Mathematics and

Mathematical

Sciences
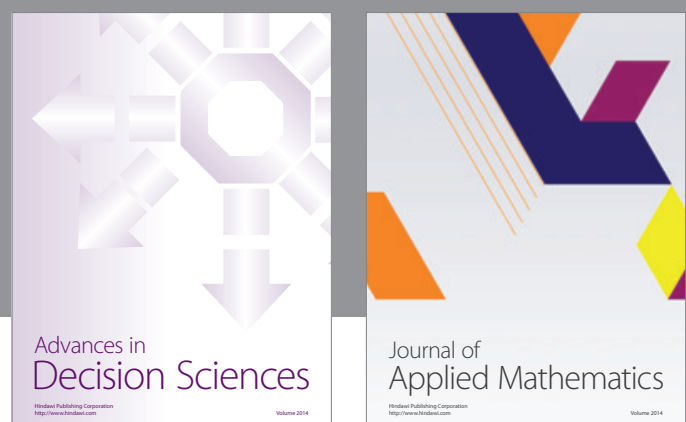

Journal of

Applied Mathematics
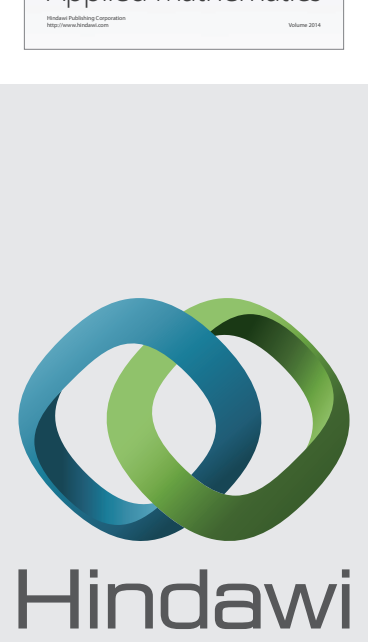

Submit your manuscripts at http://www.hindawi.com
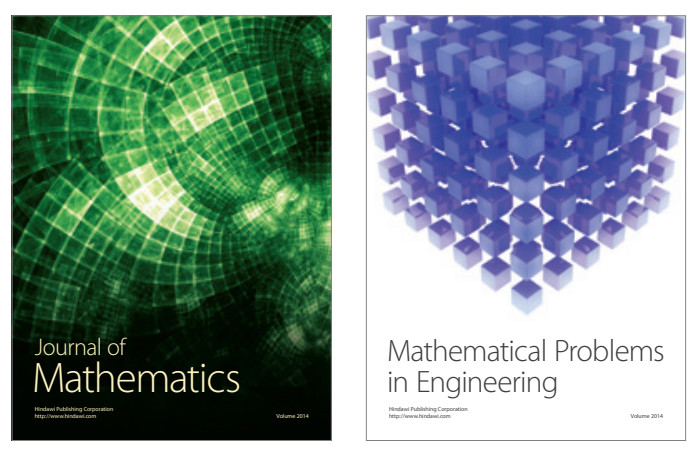

Mathematical Problems in Engineering
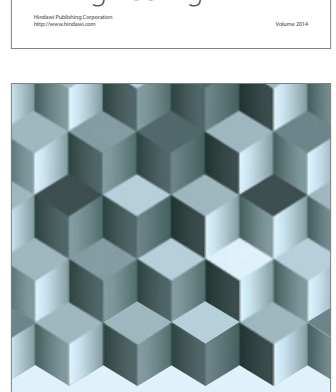

Journal of

Function Spaces
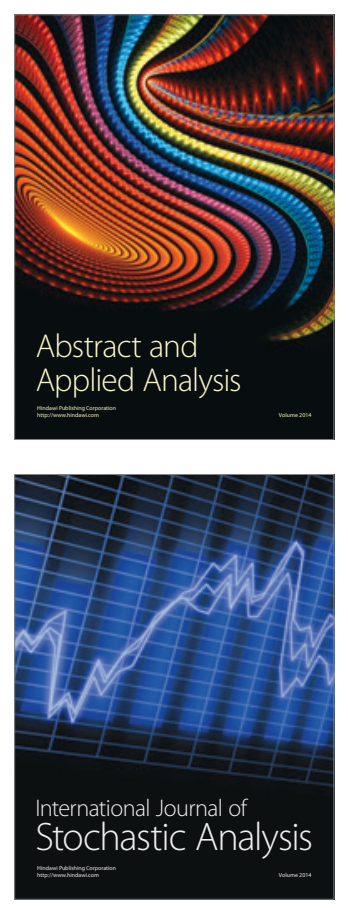

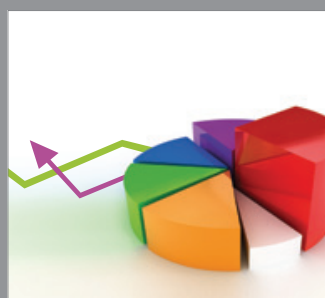

ournal of

Probability and Statistics

Promensencen
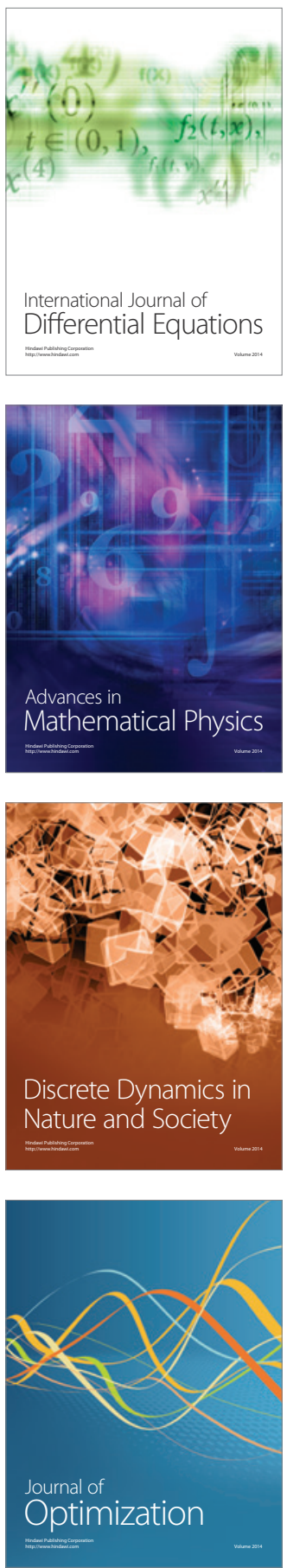